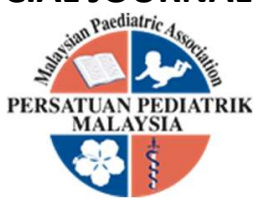

\title{
DROOLING OF SALIVA WITH DISPLACED TONGUE IN AN INFANT
}

\author{
Prempreet Kaur Manjit Singh', Shifa Zulkifli' ${ }^{2}$, Muhammad Hazim Abdul Ghafar ${ }^{2}$, Azliana Aziz ${ }^{3}$
}

\begin{abstract}
Ludwig's angina (LA) is a potentially life-threatening disease described as cellulitis of the submandibular space. There are many causes of LA with the odontogenic cause being the commonest among children and adults. In the pre-antibiotics era, LA is known to be fatal with mortality rates surpassing $50 \%$. However, with antibiotics and surgical intervention, the mortality rate has significantly reduced. We report a case of an 11-month-old infant who presented with high-grade fever, right submandibular region swelling, drooling of saliva and limited neck movement. Prompt diagnosis and airway management was required and the child underwent a successful recovery. The presentation, aetiology, management and complications are discussed.
\end{abstract}

Keywords: Ludwig's angina, odontogenic infection, infant, submandibular space

DOI: $10.51407 /$ mjpch.v28i1.158

\section{Introduction}

The superior limit of submandibular space is the mucosa of the floor of the mouth, and inferiorly by the deep investing layer of deep cervical fascia from the mandible to the hyoid bone [1]. The mylohyoid muscle runs through the middle of the submandibular space, dividing it into the superior (sublingual space) and inferior (submaxillary space) compartment $[1,2]$.

In LA, cellulitis of the floor of the mouth is characterized by induration and displacement of the tongue, which may obstruct the airway $[2,3]$.

LA is commonly reported among adult males aged between 20 to 60 with the odontogenic cause being the usual contributing factor in $70-90 \%$ of cases. However, odontogenic cause in the paediatric age group only contributes to $50 \%$ of cases $[4,5]$.

The pediatric group commonly presents with fever, neck swelling, bilateral submandibular swelling, drooling or displacement of the tongue superiorly and posteriorly which causes airway obstruction $[2,5]$. Airway obstruction can lead to fatal complications and requires prompt recognition and intervention.
Received: 27 April 2021; Accepted revised manuscript: 13 December 2021; Published online: 03 February 2022

\section{Case report}

An 11-month-old child presented with a high-grade fever for three days duration. Subsequently, he developed right submandibular swelling associated with drooling of saliva and poor oral intake. He also had limited neck movement and was less active. Clinically, the child was weak and dehydrated with persistent drooling of saliva. However, there was no stridor or noisy breathing. The child was unable to close his mouth due to the elevation of the tongue, as per Figure 1. Neck examination revealed a firm and tender right submandibular swelling extending to the right parotid region, with induration and erythematous skin as per Figure 2. Intraorally, the floor of the mouth was raised, with a small ulcer present at the sublingual surface of the right lateral

'Department of Otorhinolaryngology-Head \& Neck Surgery, Hospital Kuala Lumpur, 50586 Kuala Lumpur, Malaysia

${ }^{2}$ Department of Otorhinolaryngology-Head \& Neck Surgery, Hospital Tunku Azizah, 50586 Kuala Lumpur, Malaysia

${ }^{3}$ Department of Otorhinolaryngology-Head \& Neck Surgery, School of Medical Sciences, Universiti Sains Malaysia Health Campus, 16150 Kubang Kerian, Kelantan, Malaysia

Corresponding Author:

Dr. Azliana Aziz, Department of Otorhinolaryngology-Head \& Neck Surgery, School of Medical Sciences, Universiti Sains Malaysia Health Campus, 16150 Kubang Kerian, Kelantan, Malaysia.

Tel: +609-767 6424 Email: az_aziz@usm.my 
part of the tongue. Flexible nasoendoscopy showed medialisation of the right lateral pharyngeal wall coupled with an edematous epiglottis. The vocal cords were not visualized.

The child was electively intubated for impending airway obstruction and commenced intravenous (IV) antibiotics (ceftriaxone and metronidazole). An urgent contrast-enhanced computed tomography (CECT) neck was arranged after securing the airway, which reported inflammatory changes involving the submandibular, sublingual, parapharyngeal and retropharyngeal spaces, consistent with Ludwig's angina. Feeding was given via nasogastric tube since intubation. After 5 days of intubation, the patient was reassessed in the operation theatre which showed raised floor of mouth with the presence of induration and a sinus tract opening over the right side measuring $2 \mathrm{~cm}$ in-depth as shown in Figure $3 a$. However, at this point, the child was more stable and was successfully extubated.

The swelling subsided after the completion of ten days of intravenous antibiotics. The nasogastric tube was removed and the child could tolerate orally well. He was discharged well with oral cloxacillin which was prescribed for another fourteen days. Figure 3b shows the healing of the oral mucosa laceration wound.
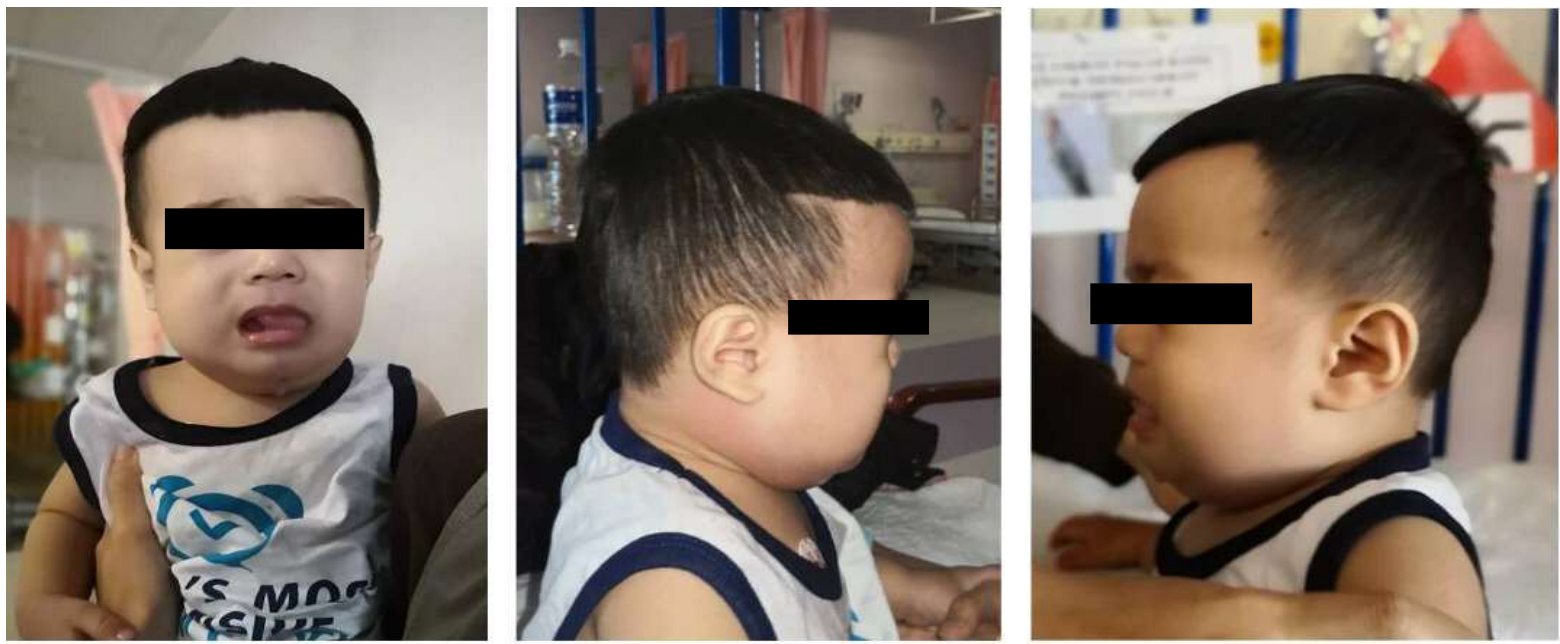

Figure 1. Images of the child upon admission. The child cannot close his mouth.

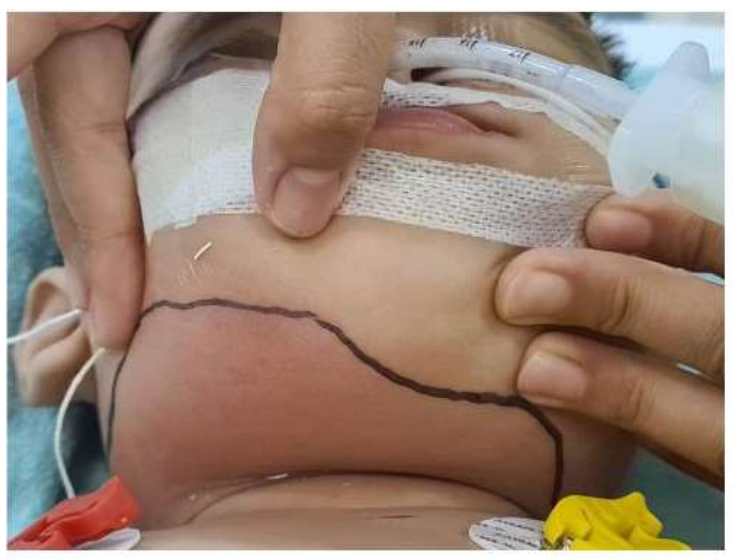

Day 1

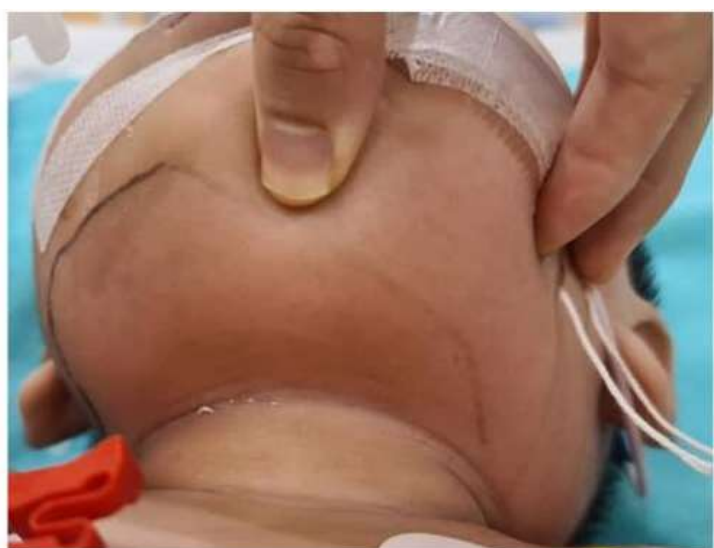

Day 2

Figure 2. Neck examination on day 1 and day. There is a right submandibular swelling extending to the right parotid region, with area of induration and erythema being demarcated. 


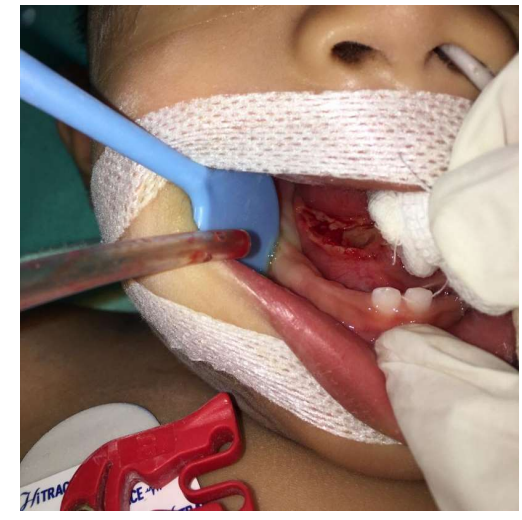

$3 a$

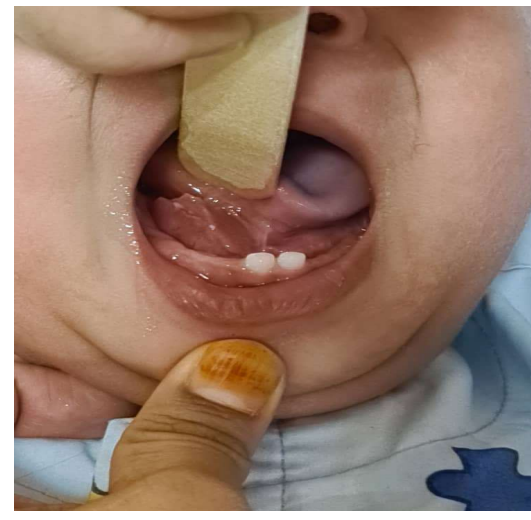

$3 b$

Figure 3. Intraoperative finding post treatment. Raised floor of mouth with the presence of induration and a sinus tract opening over the right side (3a). There is a healing of the oral mucosa laceration wound after 14 days (3b).

\section{Discussion}

The diffused inflammation of the submandibular space with progressively increasing oedema in LA causes respiratory obstruction and may lead to death [5]. It is commonly caused by poor dental hygiene usually affecting the second and third lower molar tooth among adults $[2,3,5,6]$. In children, about $50 \%$ of cases are odontogenic in origin. However, deep neck space infections secondary to upper respiratory tract infection (URTI), oral mucosa laceration or submandibular sialadenitis can also cause LA $[2,4,6]$. Apart from that, LA may also concomitantly occur together with systemic diseases such as immune deficiency and diabetes mellitus in children $[2,6]$.

Therefore, an emergency physician should always be watchful in identifying LA even in an infant who may be edentulous. A high index of suspicion is necessary to be able to diagnose LA and alert the anaesthetist and the otorhinolaryngologist to be standing by in case of airway compromise requiring airway protection as pediatric patients will deteriorate fast. Therefore, early intervention in the emergency department is crucial.

In our case, being 11 months old with only had two mandibular central incisors that could not have contributed to LA. Instead, we believe that an oral mucosa laceration found by the clinicians would have been the likely cause. This is usually caused by organisms such as aerobic-beta-hemolytic streptococcus, staphylococcus, Neisseria catarrhalis,
Escherichia coli, Hemophilus Influenza, Pseudomonas aeruginosa and anaerobic organisms [2,4]. However, since the introduction of the Hemophilus Influenza B (HiB) vaccination, the incidence caused by Hemophilus Influenza has significantly reduced [7].

One of the most feared sequelae of cellulitis of the floor of the mouth is the adjacent spread of infection causing the tongue to be posteriorly displaced, leading to life-threatening airway obstruction at the level of oral cavity and oropharynx [2,6,7]. Such patients usually present with neck swelling, the elevation of the tongue, drooling of saliva, fever and stridor $[2,6]$. This presentation usually causes a panic situation in the Emergency Room as clinicians pay the utmost attention to securing the airway as soon as possible. The mortality rate due to airway obstruction secondary to LA ranges from $54 \%$ to $60 \%$ in the pediatric population [6]. Therefore, any patient coming with audible stridor, laboured breathing or toxic look with oxygen desaturation should prompt a clinician to immediately secure the airway via endotracheal intubation, and in a worstcase scenario even via tracheostomy [8]. A study done among 29 pediatric cases of LA found that onethird of them required endotracheal intubation, whereas $7 \%$ underwent tracheostomy [3].

In this era, close observation with IV antibiotics which has sufficient bacterial and anaerobic coverage such as penicillin combined with metronidazole or clindamycin has shown tremendous improvement in managing LA $[2,6,8]$. IV steroids are also recommended to reduce 
inflammation, thus reducing oedema and cellulitis $[2,8]$. In cases of poor response to medical treatment, surgical decompression is advocated $[6,8]$.

Although the diagnosis of LA is mainly clinical, imaging modalities such as CECT or MRI can help a clinician precisely assess the extent of soft tissue inflammation thus enabling them to decide the need for surgical intervention [6]. In our case, a prompt diagnosis was achieved via an immediate CT scan and the child's airway was secured early before the occurrence of any catastrophic event. In addition, early commencement of antibiotics was also a key factor for the speedy recovery.

\section{Conclusion}

LA carries a high mortality rate among both pediatric and adult age groups. Early diagnosis at the grounds of the emergency room can be essential in early airway management. In addition, starting appropriate antibiotics can resolve LA without surgical intervention, which is becoming a trend lately. Emergency physicians and otorhinolaryngologists should be aware that in edentulous children, LA could still occur due to other non-odontogenic causes.

\section{References}

[1] Scott-Brown W, Gleeson M. Scott-Brown's Otolaryngology, Head And Neck Surgery. London: Hodder Arnold. 2008;1744.

[2] Kao JK, Yang SC. Ludwig's angina in children. J Acute Med. 2011;1:23-26.

[3] Britt JC, Josephson GD, Gross CW. Ludwig's angina in pediatric population: Report of a case and review of literature. Int J Pediatr Otorhinolaryngol. 2000;52:79-87.

[4] Papadopoulos C, Wong A, Choi J, Cheung WS. Ludwig's angina - A case report. Oral Health. $2017 ; 2$.

[5] Srirompotong S, Thumnu AS. Ludwig's angina: A clinical review. Eur Arch Otorhinolaryngol. 2003;260:401-403.

[6] Singh AP, Tanger R, Mathur V, Gupta AK. Ludwig's angina: Pediatric case report and literature review. J Mahatma Gandhi Inst Med Sci. 2018;23:89-91.

[7] Brotherton $\mathrm{H}$, Templeton K, Rowney DA and Montague ML. Ludwig's angina: Paediatric case report and literature review. Intern Med. 2014;4:174.

[8] Harrison W. Lin, MD, Allison O'Neill, MD, and Michael J. Cunningham. Ludwig's angina in pediatric population. Clin Pediatr. 2009;48:583587. 